\title{
ERRATUM
}

\section{Association of cognitive function and liability to addiction with childhood herpesvirus infections: A prospective cohort study-ERRATUM}

MICHAEL M. VANYUKOV,${ }^{a}$ VISHWAJIT L. NIMGAONKAR,${ }^{a}$ LEVENT KIRISCI, ${ }^{a}$ GALINA P. KIRILLOVA, ${ }^{a}$ MAUREEN D. REYNOLDS,${ }^{a}$ KONASALE PRASAD,${ }^{a}$ RALPH E. TARTER,${ }^{a}$ AND ROBERT H. YOLKEN ${ }^{b}$

${ }^{a}$ University of Pittsburgh; and ${ }^{b}$ Johns Hopkins University School of Medicine

doi:10.1017/S0954579417000529, published by Cambridge University Press, 19 April 2017

The critical term credible interval was mistakenly changed editorially to confidence interval in the footnote to Table 4. The entire corrected table and page are reprinted herein. We

\section{Reference}

Vanyukov, M. M., Nimgaonkar, V. L., Kirisci, L., Kirillova, G. P., Reynolds, M. D., Prasad, K., ... Yolken R. H. (2017). Association of cognitive function and liability to addiction with childhood herpesvirus infections: A sincerely regret this error and any problems or misunderstandings it may have caused.

prospective cohort study. Development and Psychopathology. Advance online publication. doi:10.1017/S0954579417000529 
Table 3. Survival (Cox proportional hazard regression) analysis or the relationships between infections (each analyzed separately) and the rate of substance use disorder development

\begin{tabular}{llccc}
\hline \hline Predictor & Sex & Hazard Ratio & $95 \%$ CI & $p$ \\
\hline HSV & Males & 1.3 & {$[0.91,1.99]$} & .141 \\
& Females & 1.7 & {$[0.91,3.01]$} & .097 \\
CMV & Males & 0.9 & {$[0.63,1.18]$} & .359 \\
& Females & 1.7 & {$[1.01,3.04]$} & .047 \\
EBV & Males & 1.3 & {$[0.95,1.80]$} & .096 \\
& Females & 2.0 & {$[1.13,3.50]$} & .016 \\
T. gondii & Males & 1.4 & {$[0.78,2.42]$} & .278 \\
& Females & 1.9 & {$[0.74,4.74]$} & .188 \\
\hline \hline
\end{tabular}

Note: Household socioeconomic status was entered as a covariate in all equations, and the analysis was stratified by ethnicity. HSV, Herpes simplex virus Type 1; CMV, cytomegalovirus; EBV, Epstein-Barr virus; T. gondii, Toxoplasma gondii; IQ, Wechsler Intelligence Scale for Childen, Third Edition, full-scale IQ; SUD, DSM-III-R diagnosis of substance use disorder.

cally, an increase), and are suggestive of a decrease by 1.6 for T. gondii.

For SUD, the results are suggestive of EBV effect in both sexes; the effect of HSV-1 in females and, somewhat weaker, in males; the effect of CMV in females, but no effect for CMV in males; and no T. gondii effect in either sex. Nevertheless, the overlapping credible intervals for the respective parameters in males and females do not indicate sex dimorphism in the relationships studied. These results largely parallel those observed in survival analysis. No infectious agentSUD diagnosis relationship is mediated by IQ (data not shown), as the indirect paths involving IQ are not significant because the direct effect of IQ on SUD risk is close to 0 (corresponding to an odds ratio close to 1.0). In addition, none of the probabilities of a higher than 0 indirect effect from the infectious agents to SUD was greater than 0.5 .

\section{Discussion}

The results of this longitudinal study are suggestive of relationships between seropositivity for common neurotropic infections HSV-1, EBV, and T. gondii in both sexes, and possibly CMV in males but not females, with lowered intelligence. Independent of these relationships, childhood EBV and HSV-1 infections in both sexes, and CMV in females, are associated with elevated risk for SUD in adulthood. This is the first study that relates childhood HHV and T. gondii infections, cognitive function, and SUD risk, and establishes the HHV-SUD risk connection. Most publications regarding cognitive impairment are in middle-aged or older individuals, whereas this study spans ages from preadolescence to adulthood. Few studies have addressed the association between HHV exposure and cognitive dysfunction. Few studies that we are aware of examined HHV seropositivity in relation to cognitive function in children (Jonker et al., 2014; Kimberlin et al., 2015; Tarter et al., 2014).

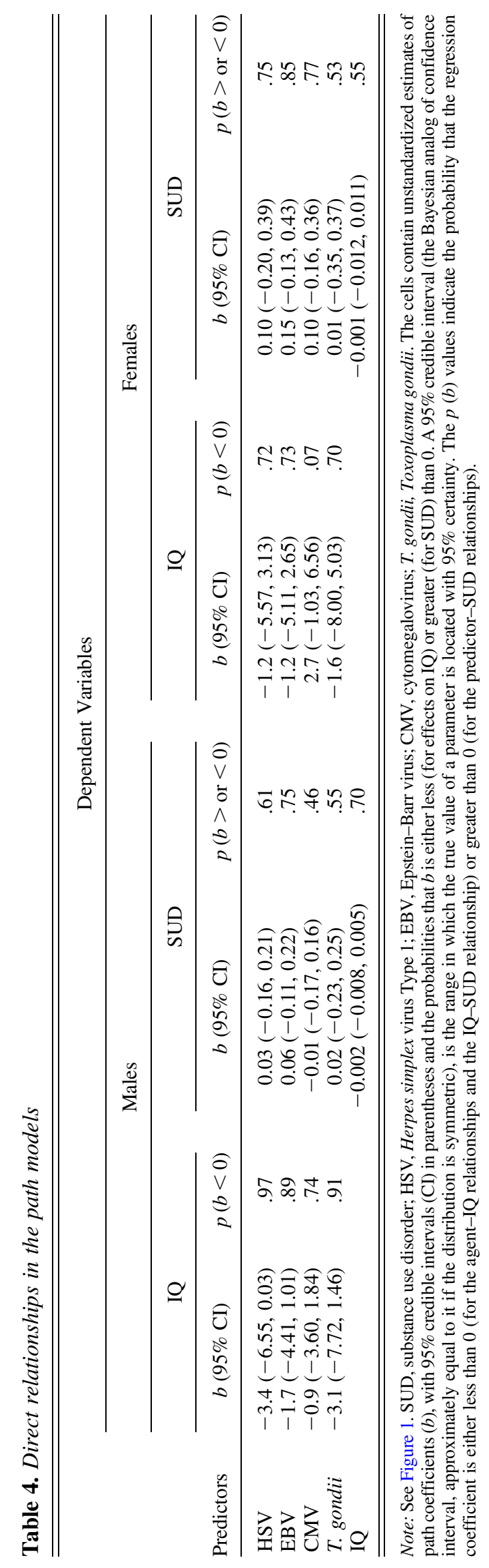

